
automation and mass data collection. These loT devices will allow businesses to develop a new range of services around their existing products and the ability to enhance service offerings.

\section{INTERNET OF THINGS AS A BUSINESS STRATEGY}

\section{Neville Auton}

\section{INTERNET OFTHINGS A BUSINESS STRATEGY}

\section{LoRa Network Topography}

The development of a Long Distance Radio (LoRa) network based Internet of Things (loT) system from idea to initial pilot deployment is presented in a case study from Otago Polytechnic based around internal space environment data collection. It was found that utilisation of state of the art new components such as LoRaWAN industrial gateways and student manufactured sensing nodes can be very beneficial for short pilot studies.

LoRa is a patented wireless data communication technology developed by Cycleo of Grenoble, France (Prashan 20।8). LoRa technology works at long distances, consumes very little power, and is strong enough to penetrate walls. This makes it an ideal solution for situations in which sensors need to send small amounts of data multiple times each day. An expected 50 billion sensing devices are expected to be in place by 2025 (Wood, 2018). Both Vodafone (Vodafone, 2019) and Spark NZ (Spark, 2018) are deploying LoRa networks to help meet the bandwidth needs of connected devices.

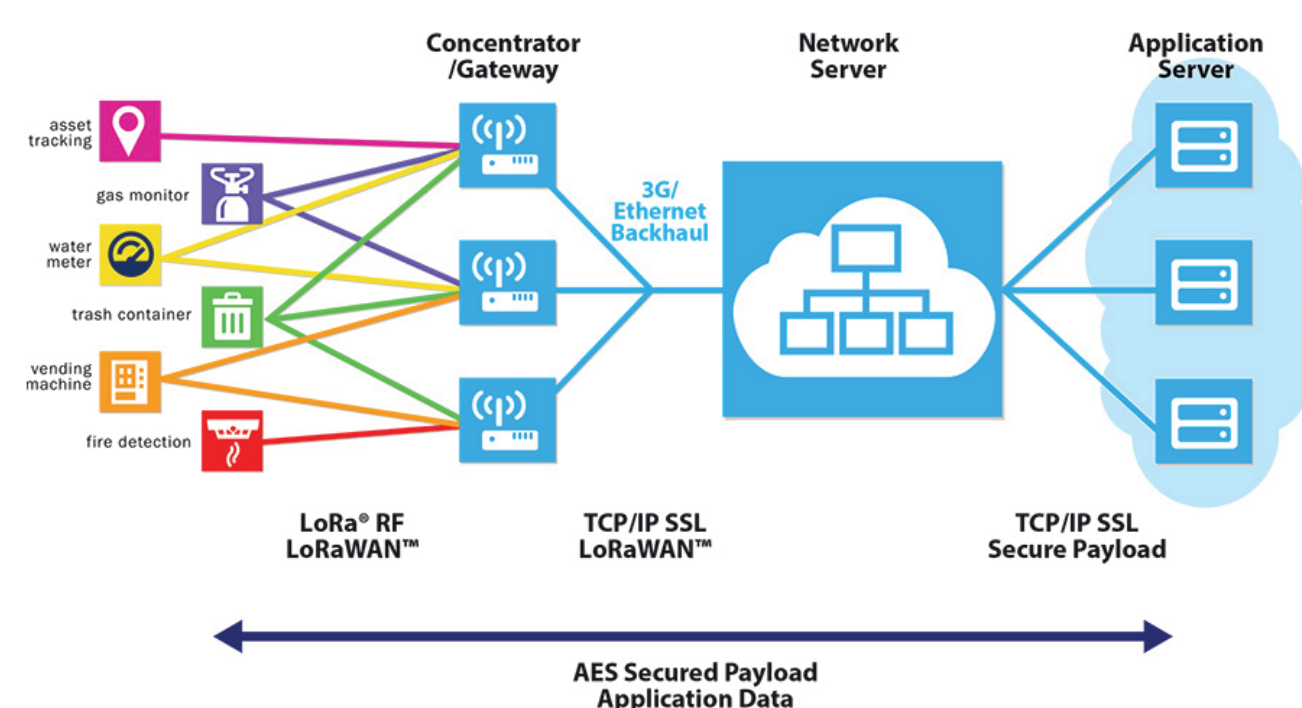

AES Secured Payload
Application Data

Figure I. LoRaTopography (wmw.researchgate.net)
Consumers will place pressure on business to have additional service or product deliverables that include loT. Smart kitchen appliances are entering the market providing consumers with added value. Smart refrigerators that Will create grocery list linked to a loca superm provid

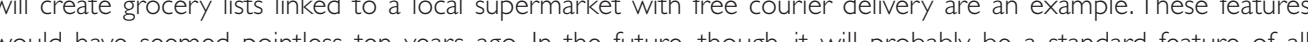
would have seemed pointless ten years ago. In the future, though, it will probably be a standard feature of all
refrigerators.

The increase in data collection from loT devices will allow detailed tracking of consumer product use behaviour. Increased data will allow technology to make product recommendations to consumers based on individual consumer preferences. As loT penetrates all aspects of business, the increase in data will change the way we target advertising and drive future smart product development.

The low cost of loT technology will see both stand alone and embedded devices increase in availability. Thus loT smart technology will transform business, providing more intelligent data and automation, and importantly it will change consumer expectations. Embedded technology will become an integral part of the product Businesses that invest in loT techno technology capability (Sniderman, Gorman, \& Hodowslky, 2016).

loT will change the way that consumers buy products with a buying cycle that is likely to become shorter. Consumers will expect a faster, more convenient service and the increasing need for instant gratification will place new demands on businesses Busnesses will need to employ smart technology so that they can keep up with consumer demands (Koesters, 2018).

loT embedded devices will allow businesses to actively monitor home appliances or business assets thereby notifying customer services when repair or replacement is required. loT reporting of fault codes for example, will allow service staff to acquire correct parts to execute a repair. loT vibration sensors will advise equipment supplies when a critical component in a compressor requires replacement This responsiveness in turn, generates improved customer service and provides new marketing opportunities (Ramaswamy, 20I6).

The tracing of inventory and assets is a major business headache. The use of loT has allowed tracking of both large and very small assets anywhere on the globe. loT asset tagging can reduce the need for manual tasks like inventory checks, thereby freeing up staff for other duties. Weighing sensors will enable for example, small part count, fluid content levels linked with location, and storage environment temperature monitoring. Additionally loT tracking has the ability to reduce the theft of inventory and assets, as well as to increase productivity across a range of corporations, small business, environmental and conservation organisations.

Understandably loT systems are transforming business efficiency and productivity, improving customer insights and customer experience, asset tracking and waste reduction. For example:

- Agricultural production, soil sensors determine irrigation need

- Smart livestock tags help manage large herds

- Electricity metering

- Street lighting control systems with automated reporting of faulty lights

- Parking meters and parking space use 
- Garbage containers will analyse and communicate fill levels

- Person trackers

Object location

Beer smart kegs monitor fill level

Environmental monitoring

- Endangered black mino tracking (or any endangered species).

\section{OTAGO POLYTECHNIC LORa ENERGY PROJECT}

The installation of a LoRa gateway at Otago Polytechnic has enabled students to research and develop LoRa senso technology to monitor temperature and humidity. These sensors transmit data every five minutes from eight roon sensors located across a floor that has wired sensors only in corridor spaces. (See Fig 2). In addition to sensor manufacture, students have created a web-based interface that allows viewing of room data on a floor plan and individual sensor temperature trending as shown below. (See Fig 3).

\section{Otago Polytechnic - Heatmap}

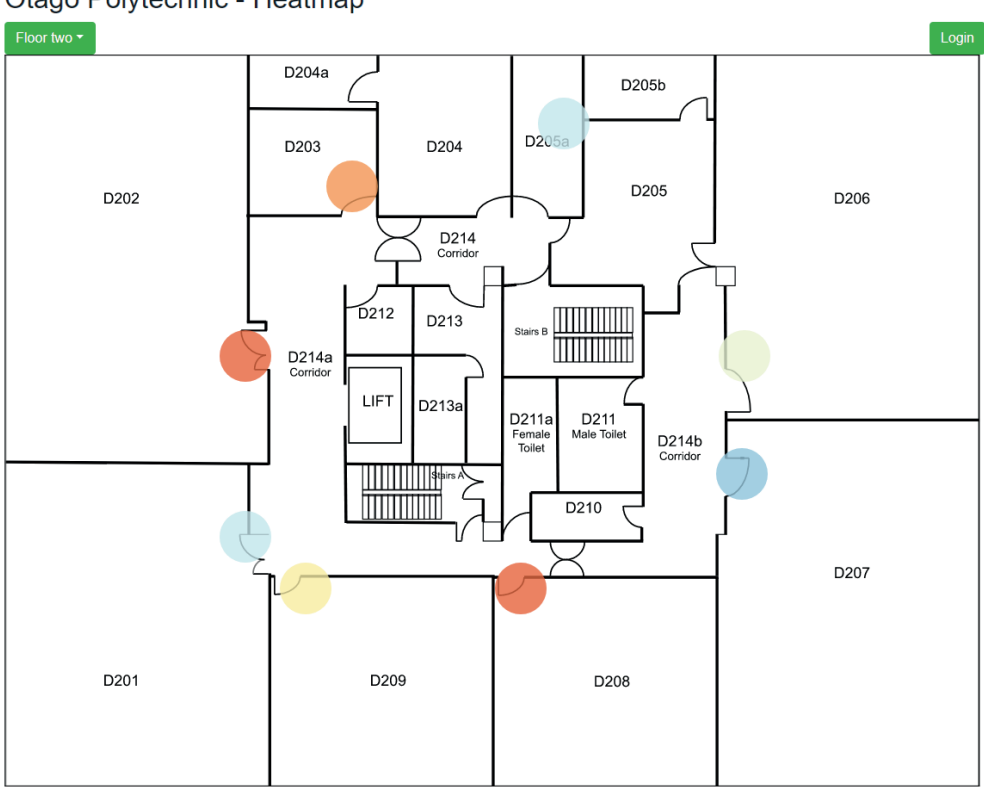

Figure 2. Floor plan with sensor locations
Otago Polytechnic - Heatmap

\section{«Back to map}

John - 2222222277771153

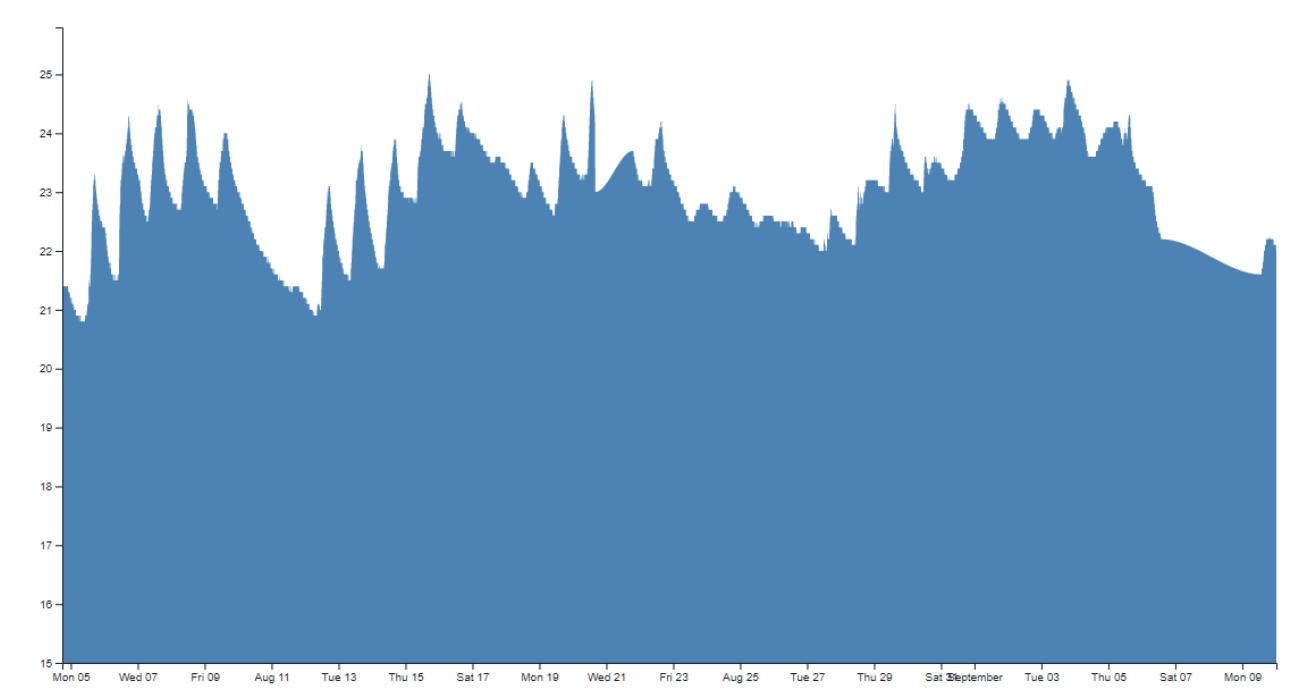

Figure 3.Temperature trending of sensor

The next generation of sensors are being enhanced to allow monitoring of temperature, humidity, $\mathrm{CO}$, light level and occupancy. With mass installation of sensors in hundreds of spaces across the campus, a full understanding of the internal environment will be gained. Space heat up and cool down timing will allow optimisation of the boiler hot water supply to the heating network. $\mathrm{CO} 2$ levels will determine if the quantity of fresh air delivery from ventilation systems is suficient for the occupancy rate of each space. Light level sensors combined with occupancy sensors will provide insight into level of spaces having lighting left on with no occupancy. Occupancy sensor data will provide an understanding of space utilisation, informing capital investment related to the need for additional space needs

Practically, LoRa offers many opportunities for student-based projects such as

- Fleet vehicle trip distance, tracking and location

- Electricity, water and LPG metering

- Irrigation requirements for lawns and garden

- Bird life count throughout campus

- Parking space availability

- Vending machine use

- Freezer/ fridge temperature monitoring

- Rubbish bin fill levels 
Although it is still at a relatively early stage, LoRa Wide Area Networks (LoRaWAN) have already found widespread acceptance among telecom companies and smart service providers across the globe. Both Vodafone and Spark in New Zealand have established networks (Spark, 2019). Capability that will allow LoRa devices to connect via secure narrow band data transfer on the cellular networks Over the next five years or so, countries including the notso-advanced nations will embrace LoRa technology due to its low cost advantages. One prediction is forecasting billions and billions of connected loT devices in operation by 2025. "Ultimately, the exact number is less relevan than the broader notion that loT adoption is ever increasing and that the connected technology is beginning to blur the boundaries between the physical and digital worlds" (Wood, 2018, p. I).

Increasingly, loT will change how business leaders, designers and manufacturers think about topics such as research and development, marketing and security. Bespoke products are replacing desire for mass produced goods; it is a serious design challenge releasing products into a marketplace where customer expectations are continually evolving. Companies already process knowledge and can use loT to enhance previous offerings of service an information delivery

One of the benefits of using loT is that it challenges organisations to think beyond the physical object and adapt to manufacturing processes to keep up with change. Businesses will utilise loT data, thus providing insights to differentiate themselves, monitor real-time developments and drive future-thinking decisions. Businesses will be able to determine if an loT solution can address a specific issue that is halting growth and how the solution will benefit their customers over time.

IoT industrial sensors can improve manufacturing processes effectively having an end to end process captured by data streams from low cost sensors. Furthermore, loT will guide product design if organisations want to meet the challenge of digital disruption, and this will in turn require loT enabled manufacturing. Data analysis for example can highlight manufacturing bottlenecks and assist to improve daily productivity. Analytics and data capture technologies will provide a move towards predictive maintenance of products and equipment (Marr, 2017).

A mass of interconnected devices and the data generated will require a well-documented loT strategic plan that provides financial gain to the bottom line. A prospective solution's functional requirements and the business core competencies will need to be aligned within a strategy. In the global data driven competitive market a comprehensive strategy will determine if an organisation is a winner or loser.

When implementing loT applications to enhance products, it is critical that organisations communicate clearly the need to build in security before deployment of solutions. Every device connecting to a business network creates risk need to b 2016) Business leaders want to be first to market Encrypting communications and values of $10 \mathrm{~T}$ data streams will provide a reduction in this risk.

The proliferation of loT technology in the future is likely to increase the number of people working from home. Many functions will be able to be carried out by home-based workers. Access to multiple loT devices in the field and within manufacturing plants will allow the development of tasks to be completed remotely Remote workers re seen as more effcient and cost effetive (Alton 2017). Future business success will be based on developing a (topation of addressing not only loT but application desion ar cross-functional collaboration is encouraged between designers and engineers.

In summary, lo $\mathrm{T}$ brings about automatic communication of data that makes way for automatic measurement and analysis of data. Business decisions will be more accurate and therefore successful, as they will be supported by real time and trending data Also, loT transformation creates a fantastic opportunity for business to completely rethink products services and development from the ground up. The world has changed Technological advances is cred
Neville Auton works at Otago Polytechnic as Energy Manager implementing energy management improvement through the development of smart devices. Neville's technical background in electronics and energy management informs innovative electronic solutions to solve energy related technical and analytical challenges, including the development of prototype low cost electricity meters, heat meters deployed across Otago Polytechnic's building infrastructure and student research.

\section{REFERENCES}

Alton, L. (2017, March 7). Are remote workers more productive than in-office workers? https://www.forbes.com/sites/

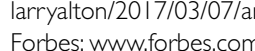

Sniderman, B., Gorman, G., \& Hodowskly, J. (2016, September 12). The design of things: Building in loT connectivity. https://wnww2 deloitte.com/insights/us/en/focus/internet-of-things/connected-products-designing-for-internet-of-things.html. Retrieved from deloitte.com/insights

Koesters, I. (2018, March 23). Connected marketing: How loT is revolutionizing customer engagement. https://wnw.digitalistmag. com/customer-experience/2018/03/23/connected-mat
Retrieved from Digitalistmag: www.digitalistmag.com

Maddox, T. (2016, July 28). Io hidden security risks: How businesses and telecommuters can protect themselves. https:// $/ \mathrm{wm}$. techrepublic.com/articleliot-hidden-security-risks-how-businesses-and-telecommuters-can-protect-themselves/ Retrieved from Techrepublic: wnw.techrepublic.com

Marr, B. (2017, May 5). Internet of things and Predictive maintenance transform the service industry. https://wnw.forbes.com/sites/ bernardmarr/2017/05/05/internet-of-things-and-predictive-maintenance-transform-the-service-industry/\#3a047c52eaf4 Betieved fom Forbes. Ww. forbes.com

Prashant, R. (2018, August 8). LPWAN, LoRa, LoRaWAN and the Internet of Things. https://medium.com/coinmonks/pwan-loralorawan-and-the-internet-of-things-aed7d5975d5d Retrieved from Medium: https://medium.com

Ramaswamy, S. (2016, June 16). Using loT data to understand how your products perform. https://hbr.org/201 6/06/using-iot-datato

Spark. (2018, September 24). Spark switches on new 'Internet of Things' network in a move to connect smart cities. https: // hmw.

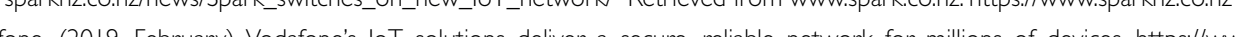

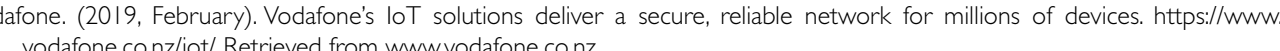
Wood, P. (2018, Feburary 21$) .5$ loT strategy principles for B2B design and manufacturing. https://wmw.iotworldtoday 